\title{
The effects of temporal modulation and spatial location on the perceived spatial frequency of visual patterns
}

\author{
LYNN MARRAN and ELIZABETH THORPE DAVIS \\ State University of New York, New York, New York
}

\begin{abstract}
The perceived spatial frequency of a visual pattern can increase when a pattern drifts or is presented at a peripheral visual field location, as compared with a foveally viewed, stationary pattern. We confirmed previously reported effects of motion on foveally viewed patterns and of location on stationary patterns and extended this analysis to the effect of motion on peripherally viewed patterns and the effect of location on drifting patterns. Most central to our investigation was the combined effect of temporal modulation and spatial location on perceived spatial frequency. The group data, as well as the individual sets of data for most observers, are consistent with the mathematical concept of separability for the effects of temporal modulation and spatial location on perceived spatial frequency. Two qualitative psychophysical models suggest explanations for the effects. Both models assume that the receptive-field sizes of a set of underlying psychophysical mechanisms monotonically change as a function of temporal modulation or visual field location, whereas the perceptual labels attached to a set of channels remain invariant. These models predict that drifting or peripheral viewing of a pattern will cause a shift in the perceived spatial frequency of the pattern to a higher apparent spatial frequency.
\end{abstract}

Several investigators have observed that changes in the perceived spatial frequency or apparent size of visual patterns may be produced by temporal modulation of the stimulus (Ansbacher, 1944; Gelb \& Wilson, 1983; Kelly, 1966; Parker, 1981, 1983; Tolhurst, 1975; Tyler, 1974) and by varying visual-field location (Davis, 1990; Davis, Yager, \& Jones, 1987; Georgeson, 1980; James, 1890; Newsome, 1972).

For example, a drifting grating viewed at the fovea has a higher perceived spatial frequency than does a foveally viewed stationary grating of the same physical spatial frequency (i.e., the foveal motion effect, FME). These shifts in perceived spatial frequency can increase up to $1 \mathrm{oc}-$ tave as the temporal frequency of the drifting grating is increased (e.g., see Parker, 1983). The effects of temporal modulation are most salient for low spatial frequency patterns.

Similarly, a stationary peripheral sinewave grating may have a higher perceived spatial frequency than does a stationary foveal pattern of the same physical spatial frequency (i.e., the location effect for stationary stimuli, LES). These shifts in perceived spatial frequency can be

This research is based on a paper submitted by Lynn Marran in partial fulfillment of the Master of Science degree requirements. The research was supported in part by NIH Grants EY05360 and EY05586, and by a Matching Funds Grant from the Research Foundation of the State University of New York to Elizabeth T. Davis. We thank Angela Brown, Ellen Ettinger, Phil Kruger, Bill Swanson, Dean Yager, and an anonymous reviewer for their helpful comments on earlier versions of the manuscript. Correspondence may be addressed to either author, Department of Visual Sciences, SUNY State College of Optometry, 100 E. 24th Street, New York, NY 10010. up to 0.6 octaves (e.g., see Davis et al., 1987). Effects of spatial location are equally salient across the range of detectable spatial frequencies. That is, for gratings whose physical spatial frequencies are below the resolution limit of the peripheral test location, the perceived spatial frequencies will change by approximately the same factor relative to their perceived spatial frequencies at the fovea.

Thus far, few, if any, investigators have examined the effect of temporal modulation on perceived spatial frequency in the periphery. For instance, few researchers have compared a drifting grating to a stationary grating when both are presented in the peripheral visual field (i.e., the peripheral motion effect, PME). Nor has the effect of visual field location on perceived spatial frequency for a drifting grating been investigated. That is, no one has compared a drifting grating presented in the periphery to a drifting grating presented at the fovea (i.e., the location effect for drifting stimuli, LED). Finally, no investigator has evaluated the combined effects of temporal modulation and spatial location on perceived spatial frequency to study possible separability of these effects on perceived spatial frequency (i.e., the combined effect, $\mathrm{CE}$ ). That is, can the difference between a drifting grating presented in the periphery compared to a stationary grating presented at the fovea (CE) be described by the sum of the motion effect (FME or PME) and the location effect for stationary stimuli (LES), as measured in octave units? If the effects are separable, then the operation of underlying mechanisms that account for the spatial location effect could be independent from the operation of mechanisms that account for the temporal modulation effect. 
In this study, we compare data on the PME to those on the FME; we also compare data on the LES to those on the LED. In addition, we present data on the combined effects of temporal modulation and of spatial location (CE), and we address the question of whether the effects of temporal modulation and visual field location on perceived spatial frequency are separable (as measured in octave units). Qualitative models based on multiple spatial frequency channels, as presented in the Discussion, may account for the data.

\section{METHOD}

\section{Stimuli}

The stimuli were vertical sinewave gratings presented on a Joyce display scope. A Gemini microcomputer produced the visual display output to the Joyce scope using digital-to-analog converters operated by an assembly-language program. Stimulus patterns were read from tables stored in memory. The display scope had a P31 phosphor, which produces light of a desaturated green hue. Mean luminance was $309 \mathrm{~cd} / \mathrm{m}^{2}$.

At the viewing distance of $114.6 \mathrm{~cm}$, the overall angular dimensions of the CRT screen were $15^{\circ}$ horizontal $\times 12.5^{\circ}$ vertical. Each grating stimulus was presented in a window created on the CRT by an assembly-language routine. Each window had a vertical extent of $1.5^{\circ}$ and a horizontal extent of $3.5^{\circ}$. The remainder of the screen was a homogenous blank field of equal mean luminance. The peripheral stimuli were always presented at $8^{\circ}$ in the superior visual field.

Stimulus duration was $540 \mathrm{msec}$ with abrupt onset and offset. We used relatively brief stimulus intervals, with pauses of $750 \mathrm{msec}$ between the stimulus intervals and pauses of several seconds between each trial, in order to minimize any spatial adaptation effects that could alter perceived spatial frequency (e.g., see Blakemore, Nachmias, \& Sutton, 1970; Blakemore \& Sutton, 1969). Brief stimulus durations with abrupt onset and offset can produce transients in the stimulus presentation. For the stimulus duration used here, however, these transients were not large enough to obscure the effect of a drifting grating on perceived spatial frequency.

To maintain a constant light-adaptation level, the display was illuminated at mean luminance both during and between stimulus presentations.

The spatial frequency of the fixed standard stimulus was $2 \mathrm{cpd}$; the standard sinusoidal grating was either stationary or drifted to the right or the left at a speed of $8.34 \%$ sec (i.e., a temporal frequency of $16.7 \mathrm{~Hz}$ ). The available comparison sinusoidal gratings ranged from 0.63 to $6.17 \mathrm{cpd}$ in equal log-unit steps of 0.004 and were always stationary. (These spatial frequency values are low enough that, even at $8^{\circ}$ in the superior visual field, no aliasing of visual patterns should occur; Coletta \& Williams, 1987.)

Contrast for all stimuli is defined as follows:

$$
\text { Contrast }=\left(L_{\max }-L_{\min }\right) /\left(L_{\max }+L_{\min }\right),
$$

where $L_{\max }$ and $L_{\min }$ are the maximum and minimum luminances of the visual pattern, respectively. Standard and comparison stimuli had a suprathreshold contrast of 0.20 .

Pattern contrast was initially calibrated using a Pritchard photometer (see Davis, Kramer, \& Yager, 1986, for details). Before each session, a "sunglasses" calibration was done to check contrast calibration. That is, a calibrated filter was positioned in front of the central bright bar of a 2-cpd squarewave grating, whose nominal contrast was $77 \%$. If the filtered bright bar did not match the perceived luminance of a neighboring, unfiltered dark bar, the contrast was adjusted and recalibrated using the Pritchard photometer.
Table 1

Experimental Conditions

\begin{tabular}{lccccc} 
& \multicolumn{2}{c}{ Location } & & \multicolumn{2}{c}{ Temporal } \\
\cline { 2 - 3 } \cline { 5 - 6 } Condition & Standard & Comparison & & Standard & Comparison \\
\hline Both & fovea & fovea & stationary & stationary \\
Fovea & fovea & fovea & drifting & stationary \\
Both & periphery & periphery & stationary & stationary \\
Periphery & periphery & periphery & drifting & stationary \\
Fovea vs. & periphery & fovea & stationary & stationary \\
Periphery & periphery & fovea & drifting & stationary \\
\hline
\end{tabular}

\section{Procedure}

There were six experimental conditions, as shown in Table 1, which can be divided into three subsets, as follows: (1) Both Fovea, in which both the standard and the comparison stimuli were presented in the fovea; (2) Both Periphery, in which both the standard and the comparison stimuli were presented in the periphery at $8^{\circ}$ in the superior visual field; and (3) Fovea versus Periphery, in which the comparison stimulus was presented in the fovea and the standard stimulus was presented at $8^{\circ}$ in the superior visual field. Within each subset there were two conditions, one in which both the standard and the comparison stimuli were stationary and the other in which the comparison stimulus was stationary and the standard stimulus was drifting.

There were four sessions for each subject, with each session including all six conditions; the order of these six conditions was randomized for each session. Each observer gave two estimates per condition per session, for a total of eight estimates per condition.

Two-interval forced-choice trials were used in our matching procedure. In the first interval of a trial, either the standard or the comparison stimulus was chosen at random and presented. In the second interval of that trial, the other stimulus was presented.

A staircase was used to match the perceived spatial frequency of a comparison stimulus to that of the standard stimulus. ${ }^{1}$ Only the spatial frequency of the comparison stimulus was varied by the staircase. Two of these staircases were randomly interleaved for a double-randomized staircase procedure. In one staircase, the spatial frequency of the initial comparison stimulus was higher than that of the standard. In the other staircase, it was lower. For drifting standard stimuli, the vertical grating drifted to the right in one staircase and to the left in the other. This presentation scheme should minimize any temporal frequency-adaptation and/or motion aftereffects (Sekuler \& Levinson, 1977).

The observer's task was to decide which of two gratings within a trial had a higher perceived spatial frequency and to indicate his or her response by pressing the appropriate lever. The observer was instructed to make this judgment by comparing the distance between two neighboring dark bars or the width of a bright-dark pair of bars for the two stimuli presented within a trial. In the Both Fovea and Both Periphery conditions, the judgment was whether the stimulus in the first or second interval had the higher spatial frequency. In the Fovea versus Periphery condition, the judgment was whether the foveal or the peripheral stimulus had the higher spatial frequency. A trial could be presented again, within the next two trials, if the observer reported that his or her eyes moved from the fixation target or that he or she had blinked during the presentation of a stimulus. ${ }^{2}$ A staircase was terminated if the step size became smaller than the criterion step size or if the observer could no longer differentiate between the standard and comparison stimuli. (These two criteria for staircase termination produced equivalent results as determined by additional testing of two naive observers.) The Both Fovea and Both Periphery stationary conditions were used as controls (foveal control, FC, and peripheral control, PC) to check both the frequency-matching staircase algorithm of the computer and each observer's ability to match spatial frequencies accurately. 
The observer adapted for 1 min to a blank, uniform screen at the mean luminance whenever the location of the comparison stimulus changed (e.g., from Both Fovea or Fovea versus Periphery to Both Periphery). The observer always fixated a small fixation target pasted on the display scope; all foveal and peripheral patterns were presented in relation to this fixation target. All stimuli were viewed monocularly with the dominant eye, as determined by a pointing task (Porac \& Coren, 1981), in a darkened room. The observer's head was held in position by a chinrest and forehead restraint. No feedback was provided.

\section{Observers}

Seven observers participated in this experiment. Six of the observers were experimentally naive. The other observer (S1) was an experienced observer who knew the purposes of the experiment. All observers had 20/20 visual acuity after any necessary refractive correction, and all were free of any ocular pathology. Observers' ages ranged from the late $20 \mathrm{~s}$ to the late $30 \mathrm{~s}$.

\section{RESULTS}

Changes in the temporal modulation and/or spatial location of a visual pattern result in significant changes in perceived spatial frequency (see Figure 1), as determined by analysis of variance for repeated measures. We examined these effects in detail, as described below, using Tukey's
HSD method or Bonferoni's test for nonorthogonal planned comparisons (Hays, 1988).

\section{Motion Effect}

The perceived spatial frequency of drifting standard stimuli was significantly higher than that of stationary comparison stimuli $[F(1,49)=199.04, p<.001]$. For theoretical reasons, we are most interested here in the FME and the PME.

Foveal motion effect (FME). The open, erect triangle at $0^{\circ}$ of eccentricity in Figure 1 shows the group data for the FME. The mean change in perceived spatial frequency for foveally viewed drifting gratings was 0.224 octaves, with a range of 0.134-0.328 octaves. This represents a significant average shift of $16.79 \%$ in perceived spatial frequency $[H S D=0.343, d f=2,36, p<.05]$, as shown in Table 2.

Peripheral motion effect (PME). The open, inverted triangle at $8^{\circ}$ of eccentricity in Figure 1 shows the group data for the PME. The mean change in perceived spatial frequency for these peripherally viewed drifting gratings was 0.147 octaves, with a range of $0.045-0.295$ octaves. This represents a significant average shift of $10.74 \%$ in perceived spatial frequency $[H S D=0.225, d f=2,36$, $p<.05$ ], as shown in Table 2 .

PERCEIVED SPATIAL FREQUENCY GROUP DATA

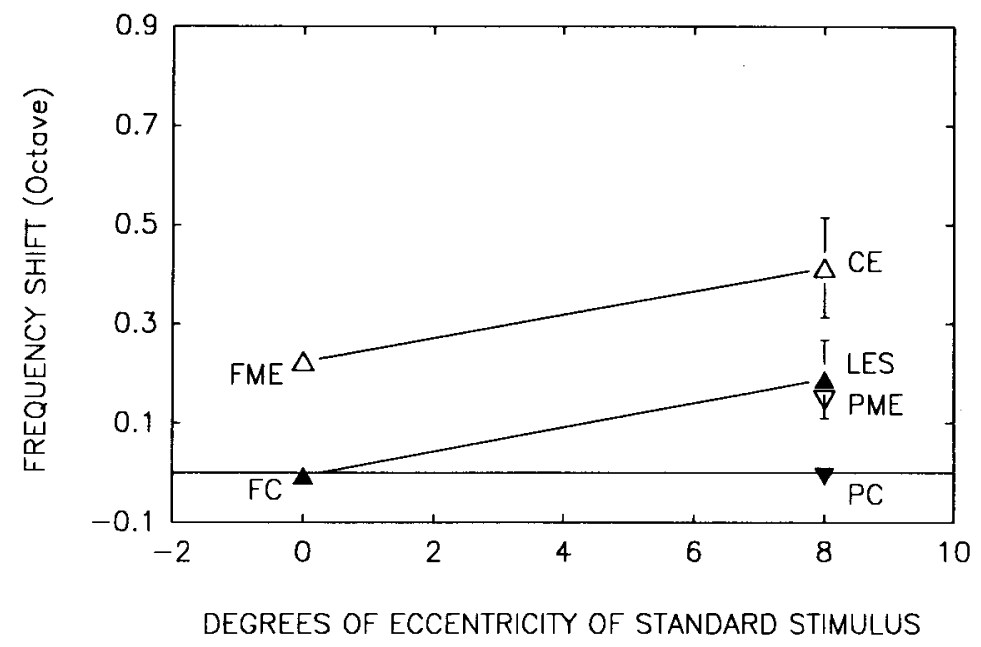

Figure 1. Group data for the effects of temporal modulation and/or spatial location on perceived spatial frequency. $F M E=$ foveal motion effect, $F C=$ foveal control, CE $=$ combined effect, LES = location effect for stationary stimuli, PME $=$ peripheral motion effect, $\mathbf{P C}=$ peripheral control, $L E D=$ location effect for drifting stimuli (CE-FME). The ordinate indicates the amount of change in perceived spatial frequency (measured in octave units), and the abscissa indicates the spatial location of the fixed standard grating (in degrees of eccentricity from the fovea). All peripheral patterns were presented at $8^{\circ}$ of eccentricity in the superior visual field. The horizontal line through zero indicates the match setting if there were no change in perceived spatial frequency (i.e., a veridical match). Each erect triangle indicates a stationary comparison stimulus presented at the fovea, and each inverted triangle indicates a stationary comparison stimulus in the periphery. Open symbols indicate that the standard stimulus was drifting, and filled symbols indicate that the standard stimulus was stationary. Each datum point is the mean of eight match settings averaged across 7 observers; the standard error bars represent \pm 1 SEM and are shown if larger than the symbol size. 
Table 2

Changes in Perceived Spatial Frequency as a Function of Motion and/or Spatial Location

\begin{tabular}{|c|c|c|c|c|c|c|c|c|}
\hline \multirow[b]{2}{*}{ Effect } & \multicolumn{7}{|c|}{ Subject } & \multirow[b]{2}{*}{$M$} \\
\hline & 1 & 2 & 3 & 4 & 5 & 6 & 7 & \\
\hline \multicolumn{9}{|l|}{ FME } \\
\hline $\begin{array}{l}\text { \% Perceived Change } \\
\text { 95\% Confidence Interval } \\
\text { PME }\end{array}$ & $\begin{array}{c}20.10 \\
17.66-22.54\end{array}$ & $\begin{array}{c}25.49 \\
18.13-32.84\end{array}$ & $\begin{array}{c}17.62 \\
14.90-20.33\end{array}$ & $\begin{array}{c}9.75 \\
6.58-12.91\end{array}$ & $\begin{array}{c}18.57 \\
8.72-28.41\end{array}$ & $\begin{array}{c}13.06 \\
8.95-17.17\end{array}$ & $\begin{array}{c}12.98 \\
7.35-18.60\end{array}$ & 16.79 \\
\hline $\begin{array}{l}\% \text { Perceived Change } \\
95 \% \text { Confidence Interval }\end{array}$ & $\begin{array}{c}22.67 \\
17.94-27.40\end{array}$ & $\begin{array}{c}12.06 \\
5.60-18.53\end{array}$ & $\begin{array}{c}19.50 \\
12.25-26.75\end{array}$ & $\begin{array}{c}6.67 \\
3.40-9.94\end{array}$ & $\begin{array}{c}3.15 \\
0.21-6.09\end{array}$ & $\begin{array}{c}3.61 \\
-1.51-8.74\end{array}$ & $\begin{array}{c}7.54 \\
2.76-12.32\end{array}$ & 10.74 \\
\hline LES & & & & & & & & \\
\hline $\begin{array}{l}\text { \% Perceived Change } \\
\text { 95\% Confidence Interval } \\
\text { LED }\end{array}$ & $\begin{array}{l}37.83 \\
32.26-43.40\end{array}$ & $\begin{array}{c}4.33 \\
-4.00-12.68\end{array}$ & $\begin{array}{c}1.18 \\
-3.30-5.66\end{array}$ & $\begin{array}{c}1.96 \\
-1.90-5.73\end{array}$ & $\begin{array}{l}35.16 \\
23.27-47.05\end{array}$ & $\begin{array}{c}17.89 \\
14.02-21.77\end{array}$ & $\begin{array}{c}-0.47 \\
-4.01-3.07\end{array}$ & 13.98 \\
\hline $\begin{array}{l}\text { \% Perceived Change } \\
\text { 95\% Confidence Interval }\end{array}$ & $\begin{array}{c}46.53 \\
33.61-59.02\end{array}$ & $\begin{array}{c}10.23 \\
4.04-17.15\end{array}$ & $\begin{array}{c}-3.86 \\
-7.70-0.02\end{array}$ & $\begin{array}{c}3.15 \\
-5.22-11.36\end{array}$ & $\begin{array}{c}30.16 \\
18.89-43.14\end{array}$ & $\begin{array}{c}10.87 \\
4.27-17.60\end{array}$ & $\begin{array}{c}-0.84 \\
-6.79-5.52\end{array}$ & 13.75 \\
\hline $\mathrm{CE}$ & & & & & & & & \\
\hline $\begin{array}{l}\text { \% Perceived Change } \\
\text { 95\% Confidence Interval } \\
\text { CE Prediction } \\
\text { (FME and LES) }\end{array}$ & $\begin{array}{c}75.99 \\
59.33-92.65\end{array}$ & $\begin{array}{l}38.32 \\
34.17-42.47\end{array}$ & $\begin{array}{c}13.07 \\
8.80-17.34\end{array}$ & $\begin{array}{c}13.20 \\
3.21-23.20\end{array}$ & $\begin{array}{c}54.33 \\
45.29-63.37\end{array}$ & $\begin{array}{c}25.34 \\
18.40-32.27\end{array}$ & $\begin{array}{c}12.03 \\
6.74-17.31\end{array}$ & 33.1 \\
\hline \% Perceived Change & $\begin{array}{c}65.53 \\
58.65-72.28\end{array}$ & $\begin{array}{c}30.92 \\
1902-4229\end{array}$ & 19.01 & 11.90 & 60.26 & 33.29 & 12.45 & \\
\hline $\begin{array}{l}\text { CE Prediction } \\
\text { (PME and LES) }\end{array}$ & $58.05-72.28$ & $9.02-42.29$ & $3.52-24.36$ & $7.00-16.67$ & $2.08-77.42$ & 39.18 & 8 & \\
\hline $\begin{array}{l}\% \text { Perceived Change } \\
\text { 95\% Confidence Interval }\end{array}$ & $\begin{array}{c}69.08 \\
60.48-77.43\end{array}$ & $\begin{array}{c}16.90 \\
6.46-26.90\end{array}$ & $\begin{array}{c}20.91 \\
12.68-28.85\end{array}$ & $\begin{array}{l}8.76 \\
3.89-13.51\end{array}$ & $\begin{array}{c}39.42 \\
27.36-50.96\end{array}$ & $\begin{array}{c}22.15 \\
15.48-28.65\end{array}$ & $\begin{array}{c}7.03 \\
1.43-12.50\end{array}$ & \\
\hline
\end{tabular}

Note-FME = foveal motion effect, PME = peripheral motion effect, LES = location effect for stationary stimuli, LED = location effect for drifting stimuli, $\mathrm{CE}=$ combined effect. All data represent the percentage of change in perceived spatial frequency, where zero represents no change.

\section{Spatial Location Effect}

Changes in the spatial location of visual patterns resulted in significant changes in perceived spatial frequency $[F(2,98)=99.11, p<.001]$. Of greatest theoretical interest to us here are the LES and the LED. (Because of potential stimulus artifacts, we were not able to measure the LED comparison directly. $)^{3}$

Location effect for stationary stimuli (LES). The closed, erect triangle at $8^{\circ}$ of eccentricity in Figure 1 shows the group data for the LES. The mean change in perceived spatial frequency for peripherally viewed stationary gratings was 0.188 octave, with a range of -0.007 to 0.463 octaves. This represents a significant average shift of $13.98 \%$ in perceived spatial frequency $[H S D=0.287$, $d f=3,36, p<.05$ ], as shown in Table 2 .

Location effect for drifting stimuli (LED). The difference between the CE and the FME, measured in octave units, is the estimate of the LED. Group data for the $\mathrm{CE}$ and the FME are shown in Figure 1 by the open erect triangles at $8^{\circ}$ and $0^{\circ}$ of eccentricity, respectively. The mean change in perceived spatial frequency for the LED was 0.186 octave. This represents a significant mean shift of $13.75 \%$ in perceived spatial frequency [HSD = $0.327, d f=3,36, p<.05]$, as shown in Table 2 .

\section{Interaction Between Motion and Spatial Location}

Analysis of variance revealed significant interaction effects between the motion and spatial location factors $[F(2,98)=3.38, p<.05]$. Although there are several possible comparisons involving interactions, we performed only two planned comparisons because these are the only comparisons of theoretical interest for our purposes.
Comparison of the FME with the PME. The FME was not significantly different in magnitude from the PME $[t(98)=0.619, p>.10]$.

Comparison of the LES with the LED. We also found that the LES and the LED were not significantly different in magnitude $[t(98)=0.034, p>.10] .{ }^{4}$ These results imply the following relationship illustrated in Figure 1: A diagonal line connecting the FME and the CE by definition represents the change in perceived spatial frequency due to the LED. This diagonal line should be parallel to a diagonal line connecting the FC and the LES, which represents the change in perceived spatial frequency due to the LES, if the LES and the LED are of equal magnitude.

\section{Comparison of Predictions with the Data}

If the effect due to motion is separable from the effect due to spatial location of the visual pattern, one can predict the perceived spatial frequency of a peripherally viewed drifting pattern as compared with a foveally viewed stationary comparison pattern (i.e., the $\mathrm{CE}$ ). The means and 95\% confidence intervals of the predicted and actual combined effects are shown in Table 2. For most observers, the confidence intervals for the predicted and actual combined effects overlap. These predictions were made as described below.

The predicted CE is the LES multiplied by the motion effect [i.e., $F(A B)=F(A) F(B)$, where $F(\cdot)=1.0$ indicates no change and $F(\cdot)=1.20$ indicates a $20 \%$ change in perceived spatial frequency].

Our estimation of the spatial location effect is the LES. However, we previously discussed two different estimations of the motion effect, the FME and the PME. Which 
of these is the most appropriate estimate to use? One might expect that if motion effects and spatial location effects are separable, then it should not matter which motion estimate is used. Indeed, the group data show that there was no significant difference in the magnitudes of the FME and PME. The individual sets of data also show that, for most subjects, there was no significant difference in the magnitudes of the FME and the PME.

A Wilcoxon signed-rank test was used to determine if one motion estimate is a better predictor than the other, evaluated across individual sets of data. Not surprisingly, neither the FME nor the PME is a significantly better predictor $(p=.109)$.

Note that if the spatial location effect and the motion effect are in octave or log units, then separability of effects implies that the shifts obtained for each can be added to predict the CE [e.g., $\left(2^{a}\right)\left(2^{b}\right)=2^{(a+b)}$ ]. For example, suppose the FME were used as the estimate for the motion effect and the LES for the spatial location effect. If the effects are separable, then one would expect a line connecting the FME to the CE in Figure 1 to be parallel to a line connecting the FC to the LES. (Also note, as previously discussed, that this relationhip implies that the LED should not significantly differ in magnitude from that for the LES, when measured in octave units.) The PME could also be used to estimate the motion effect and does not differ significantly from the FME. However, partially because of the spatial configuration of Figure 1, the above intuitive relationship of parallel lines is not evident when the PME is used.

\section{Control Conditions}

In 13 of the 14 data estimates from all observers for the two control conditions (FC and PC), the match comparison spatial frequency was not significantly different from the physical spatial frequency of the standard, as determined from the $95 \%$ confidence intervals for the individual data. Furthermore, none of the 14 control match spatial frequency settings differed significantly from the standard spatial frequency as determined by the $99 \%$ confidence intervals. Thus, the observers were able to match perceived spatial frequency accurately.

\section{DISCUSSION}

\section{Motion Effect}

Empirical results. A drifting grating often has a higher perceived spatial frequency than does a stationary grating when both are viewed at the same visual field location. This result has previously been reported for foveally and parafoveally viewed low spatial frequency drifting gratings (e.g., Parker 1981, 1983; Virsu, Nyman, \& Lehtio, 1974), counterphase-modulating gratings (e.g., Gelb \& Wilson, 1983; Kelly 1966, 1981; Parker 1981, 1983; Tyler, 1974; Virsu et al., 1974), and stimuli of varying durations (e.g., Kulikowski, 1975; Tynan \& Sekuler, 1974). However, few, if any, researchers have inves- tigated the effects of motion in the peripheral visual field, as we did here.

Overall, the group mean data show no significant difference in the magnitude of the foveal and peripheral motion effects (see Figure 1).

Qualitative model. The predictions of a mechanistic "selectivity-shift" model based on changes in the spatial frequency tuning of multiple spatial frequency channels, but an invariant set of perceptual labels (Watson \& Robson, 1981), are consistent with these data. The underlying substrate of multiple spatial frequency channels may be mechanisms whose receptive-field profiles have excitatory centers and inhibitory surrounds (or vice versa). The inhibitory portion may react more sluggishly (i.e., have a longer time constant) than the excitatory portion, so that under transient stimulation, the inhibitory portion has relatively little effect (e.g., see Derrington \& Lennie, 1982; Enroth-Cugell \& Lennie, 1975; Maffei, Cervetto, \& Fiorentini, 1970). Thus, transient stimulation, which is characteristic of counterphase modulating or drifting gratings, would reduce or eliminate the inhibitory contribution of a mechanism's response (Tynan \& Sekuler, 1974; Virsu \& Nyman, 1974). This effectively reduces the optimal spatial frequency to which the mechanism will respond. That is, these mechanisms demonstrate a lack of spatiotemporal separability within a given mechanism.

If the perceptual spatial frequency labeling remains invariant but the spatial frequency characteristics of the mechanism have shifted to lower physical spatial frequencies (e.g., see Georgeson, 1980), then a drifting grating will be perceived as having a higher spatial frequency than a stationary grating of the same physical spatial frequency. That is, suppose a mechanism has a peak sensitivity to a stationary pattern of $2 \mathrm{cpd}$. When stimulated by a drifting pattern, the peak sensitivity will be shifted to a lower spatial frequency (e.g., 1.5 cpd), but its perceptual label remains fixed at $2 \mathrm{cpd}$. Thus, a drifting grating of lower spatial frequency (e.g., $1.5 \mathrm{cpd}$ ) will match the perceived spatial frequency of a 2-cpd stationary grating. Our results are consistent with this interpretation.

Modifications of the model. The selectivity-shift model may not hold in its simplest form, as described above in the qualitative model, in which one assumes that detection and perception occur at the same level (e.g., see Parker, 1981, 1983). Psychophysical mechanisms at a fixed location seem to have spatial and temporal separability at the level of detection (e.g., Graham, 1972; Parker, 1981). For instance, Parker (1981) examined subjects' contrast threshold for stationary gratings before and after adaptation to a counterphase modulating grating. According to predictions based on the selectivity-shift model, the maximum contrast threshold elevation should occur at the apparent spatial frequency rather than the physical spatial frequency of the adapting stimulus. However, the greatest threshold elevation occurred at the physical spatial frequency of the adapting stimulus. In contrast, in another experiment designed to examine the effects of adaptation 
to a temporally modulated stimulus on the perceived spatial frequency shift phenomenon (Blakemore \& Sutton, 1969), Parker found that the spatial frequency shift was centered around the apparent spatial frequency of the adapting drifting gratings, not the physical spatial frequency of the adapting stimulus. The results of Parker's perceived spatial frequency shift experiment are in accord with the predictions of the selectivity-shift model.

On the basis of these adaptation results, Parker (1981, 1983) suggested a two-stage hierarchical model similar to earlier models suggested by Klein, Stromeyer, \& Ganz (1974) and by Heeley (1979). At a lower stage, where detection occurs, there are spatiotemporal mechanisms that demonstrate spatial and temporal separability. However, at a higher stage, where size perception occurs, spatiotemporal characteristics of mechanisms are no longer separable.

We maintain that alternative hypotheses are also possible. Perhaps there are separate and independent parallel pathways. One pathway, primarily responsible for detection of spatiotemporal stimuli, may have only mechanisms that manifest spatiotemporal separability. Another pathway (or pathways), primarily responsible for processing form and/or motion information, may have at least some mechanisms that lack spatiotemporal separability. In this regard, physiological findings in the cat may be instructive. Although Tolhurst and Movshon (1975) found spatiotemporal separability in the function of striate cortical cells, others (e.g., Derrington \& Lennie, 1982; EnrothCugell \& Lennie, 1975; Maffei et al., 1970) have found spatiotemporal inseparability at lower level cells (e.g., retinal ganglion cells). These lower level cells, instead of providing input to the striate cortex (Area 17), may provide input to extrastriate cortical cells (Area 18), where Bisti, Carmignoti, Galli, and Maffei (1985) also found a lack of separability in the spatiotemporal characteristics of cells.

A more probable model may be a hybrid of the above two models. Perhaps there are parallel pathways that can communicate with each other and that also possess a hierarchical ordering of functions. This version would be consistent with DeYoe and Van Essen's (1988) report that there are interconnections between various parallel pathways of the magnocellular and parvocellular systems in the monkey, and that these pathways also demonstrate a hierarchical ordering of function.

It is also possible that the individual psychophysical mechanisms possess spatiotemporal separability, but that acting together they behave in a manner that produces overall spatiotemporal inseparability. The spatiotemporal contrast-sensitivity functions for detection of visual patterns (e.g., see Robson, 1966) could be an example of individual psychophysical mechanisms whose individual operation is separable in space and time but whose combined operation produces an overall lack of spatiotemporal separability. The details of such a model must be developed further to account for changes in perceived spatial frequency as a function of temporal modulation.

\section{Spatial Location Effect}

Empirical results. Overall, the perceived spatial frequency of peripherally viewed patterns was significantly higher than that of foveally viewed patterns for both stationary and temporally modulated gratings (see Figure 1). Other investigators (Davis, 1990; Davis et al., 1987; Georgeson, 1980; James, 1890; Newsome, 1972) have reported that stationary patterns often appear smaller (or of higher apparent spatial frequency) when viewed in the periphery than when viewed foveally. These results have now been extended to include foveally versus peripherally viewed temporally modulating patterns.

Qualitative model. A model based on changes in receptive-field sizes of psychophysical mechanisms as a function of visual field eccentricity, but an invariant set of perceptual labels, may account for these data (Davis, 1990; Davis et al., 1987; Georgeson, 1980). That is, the spatial frequency tuning of peripheral mechanisms is lower than that of foveal psychophysical mechanisms (Graham, Robson, \& Nachmias, 1978; Swanson \& Wilson, 1985; Wilson \& Bergen, 1979). If the perceived spatial frequency labels attached to a set of mechanisms do not change as a function of eccentricity, but the set of peripheral mechanisms is tuned to lower spatial frequencies than is the foveal set, then a grating viewed in the periphery will have a higher apparent spatial frequency than a grating of the same physical spatial and temporal frequency viewed in the fovea. Overall, our results are consistent with this interpretation.

Modifications of the model. There are other mechanism characteristics that change as a function of visual field eccentricity and which can also affect the appearance of visual patterns. For instance, contrast sensitivities of peripheral mechanisms are lower than those of foveal mechanisms (Daitch \& Green, 1969; Davis, 1990; Lundh, Lennerstrand, \& Derefeldt, 1983; Rijsdijk, Kroon, \& van der Wildt, 1980; Robson \& Graham, 1981; Wilson \& Bergen, 1979) for stimulus patches of fixed vertical and horizontal extents. Calculations show that the effect of reduced sensitivity in the periphery is opposite that predicted from increased receptive-field sizes (Davis, 1990). Thus, reduced sensitivity operates to reduce the perceived spatial frequency of peripheral patterns. The opposing effects of increased receptive-field sizes and reduced sensitivities of peripheral mechanisms (compared with the set of foveal mechanisms) may result in little or no net change in perceived spatial frequency between the fovea and the periphery.

There are certainly individual differences both in receptive-field scaling (Davis, 1990; Swanson \& Wilson, 1985) and in reduced sensitivity of peripheral psychophysical mechanisms relative to foveal mechanisms (Davis, 1990; Rijsdijk et al., 1980). These individual differences could account for the individual differences found in the perceived spatial frequency shifts between the fovea and the periphery. The fact that the same observers who showed the largest changes for temporally modulating gratings as a function of visual field location 
(LED) also showed the largest changes for stationary gratings (LES), as shown in Table 2 , is consistent with the above interpretation. Moreover, the fact that the observers who showed the smallest changes for stationary gratings also showed the smallest changes for temporally modulating gratings is also consistent with the above interpretation.

\section{Comparisons of Temporal and Spatial Location Effects}

Combined effects of motion and location are typical of what may happen in a complex, real visual scene. The combined effect (i.e., the perceived spatial frequency of a drifting, peripherally viewed stimulus compared with a stationary, foveally viewed stimulus) should be predictable from the motion effect and the spatial location effects if the two effects are separable. That is, separability implies that the operation of underlying mechanisms that account for the location effect is independent of those which account for the motion effect. Here the emphasis is on the magnitudes of the effects themselves, however, rather than on details of the underlying psychophysical mechanisms. If the effects are separable (albeit the motion effect and the location effect may each be relatively small), the combined effect can be quite large (up to 0.8 octaves in our data).

The spatial location effect for stationary stimuli is the only estimate of the spatial location effect. Recall that an estimate of the motion effect could be either the foveal motion effect or the peripheral motion effect. Because most of our subjects (6 out of 7) showed no significant difference between the magnitudes of the foveal and peripheral motion effects, overall it should not matter which effect is used to estimate the motion effect. In fact, the Wilcoxon signed-rank test shows that, overall, neither the foveal nor the peripheral motion effect is a significantly better predictor for the combined effect.

The combined effects are reasonably predicted by the sum of the motion effect and the spatial location effect (measured in octave units), both for the group data and for each individual's set of data. (See Table 2 for means and $95 \%$ confidence limits of obtained and predicted combined effects.) Thus, both the group average results and the results of most of our observers are consistent with the concept of separability for the spatial location effect and the motion effect on perceived spatial frequency.

\section{SUMMARY}

Both temporal modulation and visual field location are known to affect perceived spatial frequency, but previous studies have not examined changes in perceived spatial frequency when these two conditions are combined. Our group data, as well as most of the individual sets of data, suggest that the two effects are separable (i.e., additive in octave or log units). This suggests that the operation of underlying mechanisms that cause one effect is independent of those that cause the other effect. Moreover, although each effect may be relatively small, the combined effects (typical of what may happen in a real visual scene) are quite large and worthy of study.

Two qualitative models suggest possible explanations for these effects. Both models assume that although changes in temporal modulation or visual field location result in changes of the receptive-field sizes of sets of underlying psychophysical mechanisms, the perceptual labels attached to those channels remain invariant.

\section{REFERENCES}

Ansbacher, H. L. (1944). Distortion in the perception of real movement. Journal of Experimental Psychology, 34, 1-23.

Bisti, S., Carmignoti, G., Galli, L., \& Maffei, L. (1985). Spatialfrequency characteristics of neurones of area 18 in the cat: Dependence on the velocity of the visual stimulus. Journal of Physiology, 359, 259-268.

Blakemore, C., Nachmias, J., \& Sutton, P. (1970). The perceived spatial frequency shift: Evidence for frequency-selective neurones in the human brain. Journal of Physiology, 210, 727-750.

Blakemore, C., \& Sutton, P. (1969). Size adaptation: A new aftereffect. Science, 166, 245-247.

Coletta, N. J., \& Williams, D. R. (1987). Psychophysical estimate of extrafoveal cone spacing. Journal of the Optical Society of America, 4, 1503-1513.

Daitch, J. M., \& Green, D. G. (1969). Contrast sensitivity of the human peripheral retina. Vision Research, 9, 947-952.

DAvIs, E. T. (1990). Modeling shifts in perceived spatial frequency between the fovea and the periphery. Journal of the Optical Sociery of America, 7, 286-296.

Davis, E. T., Kramer, P., Yager, D. (1986). Shifts in perceived spatial frequency of low-contrast stimuli: Data and theory. Journal of the Optical Society of America, 3, 1189-1202.

Davis, E. T., YAGER, D., JoneS, B. J. (1987). Comparison of per ceived spatial frequency between the fovea and the periphery. Journal of the Optical Society of America, 4, 1606-1611.

Derrington, A. M., \& LenNie, P. (1982). The influence of temporal frequency and adaptation level on receptive field organization of retinal ganglion cells in cat. Journal of Physiology, 333, 343-366.

DeYoe, E. A., \& Van Essen, D. C. (1988). Concurtent processing streams in monkey visual cortex. Trends in Neuroscience, 11, 219-226.

Enroth-Cugell., C., \&ennie, P. (1975). The control of retinal ganglion cell discharge by receptive field surrounds. Journal of Physiology, 247, 551-578.

Gelb, D., \& WiLson, H. R. (1983). Shifts in perceived size as a function of contrast and temporal modulation. Vision Research, 23, 71-82.

Georgeson, M. A. (1980). Spatial frequency analysis in early visual processing. Philosophical Transactions of the Royal Society of London, B290, 11-21.

GrahaM, N. (1972). Spatial frequency channels in the human visual system: Effects of luminance and pattern drift rate. Vision Research, $12,53-68$.

Graham, N., Robson, J. G., \& Nachmias, J. (1978). Grating summation in fovea and periphery. Vision Research, 815-827.

Hays, W. L. (1988). Statistics (4th ed.). New York: Holt, Rinehart \& Winston.

HeELEY, D. W. (1979). A perceived spatial frequency shift at orientations orthogonal to adapting gratings. Vision Research, 19, 1229-1236.

James, W. (1890). Principles of Psychology (Vol. 2). London: Macmillan.

KELLY, D. H. (1966). Frequency doubling in visual responses. Journal of the Optical Society of America, 56, 1628-1633.

KeLLY, D. H. (1981). Nonlinear visual responses to flickering sinusoidal gratings. Journal of the Optical Society of America, 71, 1051-1055.

Klein, S., Stromeyer, C. F., \& Ganz, L. (1974). The simultaneous spatial frequency shift: A dissociation between the detection and perception of gratings. Vision Research, 14, 1421-1432. 
KuLIKowsKı, J. J. (1975). Apparent fineness of briefly presented gratings: Balance between movement and pattern channels. Vision Research, 15, 673-680.

Lundh, B. L., Lennerstrand, G., \& Derefeldt, G. (1983). Central and peripheral normal contrast sensitivity for static and dynamic sinusoidal gratings. Acta Ophthalmologica, 61, 171-182.

Maffei, L., Cervetto, L., \& Fiorentini, A. (1970). Transfer characteristics of excitation and inhibition in cat retinal ganglion cells. Joumal of Neurophysiology, 33, 276-284.

Murphy, B. J., Kowler, E., \& Steinman, R. M. (1975). Slow oculomotor control in the presence of moving backgrounds. Vision Research, 15, 1263-1268.

Newsome, L. R. (1972). Visual angle and apparent size of objects in peripheral vision. Perception \& Psychophysics, 12, 300-304.

PARKER, A. (1981). Shifts in perceived periodicity induced by temporal modulation and their influence on the spatial frequency tuning of two aftereffects. Vision Research, 21, 1739-1747.

PARKer, A. (1983). The effects of temporal modulation on the perceived spatial structure of sine-wave gratings. Perception, 12, 663-682.

PORAC, C., \& COREN, S. (1981). Lateral preferences and human behavior. New York: Springer.

Rijsdijk, J. P., Kroon, J. N., \& Van der Wildt, G. J. (1980). Contrast sensitivity as a function of position on the retina. Vision Research, 20, 235-241.

RoBson, J. G. (1966). Spatial and temporal contrast-sensitivity functions of the visual system. Journal of the Optical Society of America, 56, 1141-1142.

Robson, J. G., \& Graham, N. (1981). Probability summation and regional variation in contrast sensitivity across the visual field. Vision Research, 21, 409-418.

SEKULER, R., \& LEVINSON, E. (1977). The perception of moving targets. Scientific American, 236, 60-73

SWANSON, W., \& Wilson, H. R. (1985). Eccentricity dependence of contrast matching and oblique masking. Vision Research, 25, $1285-1295$

ToLhuRst, D. J. (1975). Illusory shifts in spatial frequency caused by temporal modulation. Perception, 4, 331-335.

Tolhurst, D. J., \& Movshon, J. A. (1975). Spatial and temporal contrast sensitivity of striate cortical neurones. Nature, 257, 674-675.

TYLER, C. W. (1974). Observations on spatial-frequency doubling. Perception, 3, 81-86.

Tynan, P., Sexuler, R. (1974). Perceived spatial frequency varies with stimulus duration. Journal of the Optical Society of America, 64, 1251-1254.

Virsu, V., \&yman, G. (1974). Monophasic temporal modulation increases apparent spatial frequency. Perception, 3, 337-353.

Virsu, V., Nyman, G., Lehtio, P. K. (1974). Diphasic and polyphasic temporal modulation increases apparent spatial frequency. Perception, 3, 323-336.

WATSON, A. B., RoBson, J. G. (1981). Discrimination at threshold: Labeled detectors in human vision. Vision Research, 21, 1115-1122.
WILSON, H. R., \& BERGEN, J. R. (1979). A four mechanism model for threshold spatial vision. Vision Research, 19, 19-32.

\section{NOTES}

1. Each staircase was independent of the other staircase, but both were governed by the following rules: (a) The initial step size was the maximum step size used $\left(0.128 \log _{10}\right.$ units). (b) If the subject reported that the comparison stimulus had a higher spatial frequency than the standard, then the comparison spatial frequency was decreased; if the subject reported that the comparison stimulus had a lower spatial frequency, then the comparison spatial frequency was increased. (c) Each reversal of direction resulted in halving the step size. (d) More than two traversals in the same direction resulted in doubling the step size. (e) When a minimum step size $\left(0.004 \log _{10}\right.$ units) corresponding to the exit criterion was reached, the spatial frequency of the comparison stimulus was recorded as the match to the standard, and that staircase was terminated.

2 . If an observer had pursuit eye movements during the presentation of the drifting grating, this could reduce or eliminate any shifts in perceived spatial frequency for the foveal and peripheral motion effects. However, a fixation target was always provided, the observers were told to maintain fixation and reported doing so, and each observer's fixation was periodically monitored by the experimenter. In fact, Murphy, Kowler, and Steinman (1975) found that when a fixation point is provided, observers can hold fixation quite well while viewing small patches of drifting grating.

3. The foveal comparison stimulus was stationary rather than drifting because varying the spatial frequency of a drifting stimulus can result in physical changes due to electronic digitization of the stimulus display. That is, the temporal frequency of the drifting stimulus and even the direction of motion may be changed by varying the spatial frequency of a drifting grating. Thus, it was necessary to compute the LED indirectly, by subtracting the FME from the $\mathrm{CE}$, both in octave units, as described in the text.

4. The analysis of variance and most of the planned and post hoc statistical comparison tests were performed on the match spatial frequency values of the comparison stimuli. However, for the figures and some of the comparison tests (relevant to theoretical considerations discussed later), the perceived spatial frequency shifts are represented in octave units. Specifically, the comparisons of the LED with the LES were performed using octave values. This was necessary because the base frequencies for the LES and the LED were different. In contrast, for the motion effect comparisons, the base frequencies for the FME and the PME were both 2 cpd; thus, the analysis performed on match spatial frequency results are similar to those performed on octave values.

(Manuscript received May 24, 1989; revision accepted for publication November $21,1989$. 